\title{
Use of an electric ear syringe in the emergency department
}

\author{
Ian Jones, Chris Moulton
}

\begin{abstract}
Objectives-To introduce an electric ear syringe into an emergency department and evaluate its use in the removal of foreign bodies from the external auditory canal.
\end{abstract}

Methods-Report of the use of a new device (the Propulse electric ear syringe) in an emergency department with a retrospective audit of the management of all cases of aural foreign bodies during one year.

Results-Almost $60 \%$ of the 84 patients who presented with foreign bodies in the auditory canal were children. Staff chose to treat over half of all patients with aural foreign bodies with the electric ear syringe. The overall success rates for the removal of the foreign bodies were: electric syringe, $93 \%$ in adults and $88 \%$ in children and instrumentation, $68 \%$ in adults and $50 \%$ in children.

Conclusions-Irrigation with an electric ear syringe is an effective method of removing foreign bodies from the external auditory canal. The device tested was safe, inexpensive, easy to use, and readily acceptable to both adults and children. The availability of an electric ear syringe in an emergency department can avoid the need for specialist referral and the subsequent removal of foreign bodies under general anaesthesia.

$(\Im$ Accid Emerg Med 1998;15:327-328)

Keywords: foreign bodies; electric ear syringe

Accepted for publication 24 April 1998

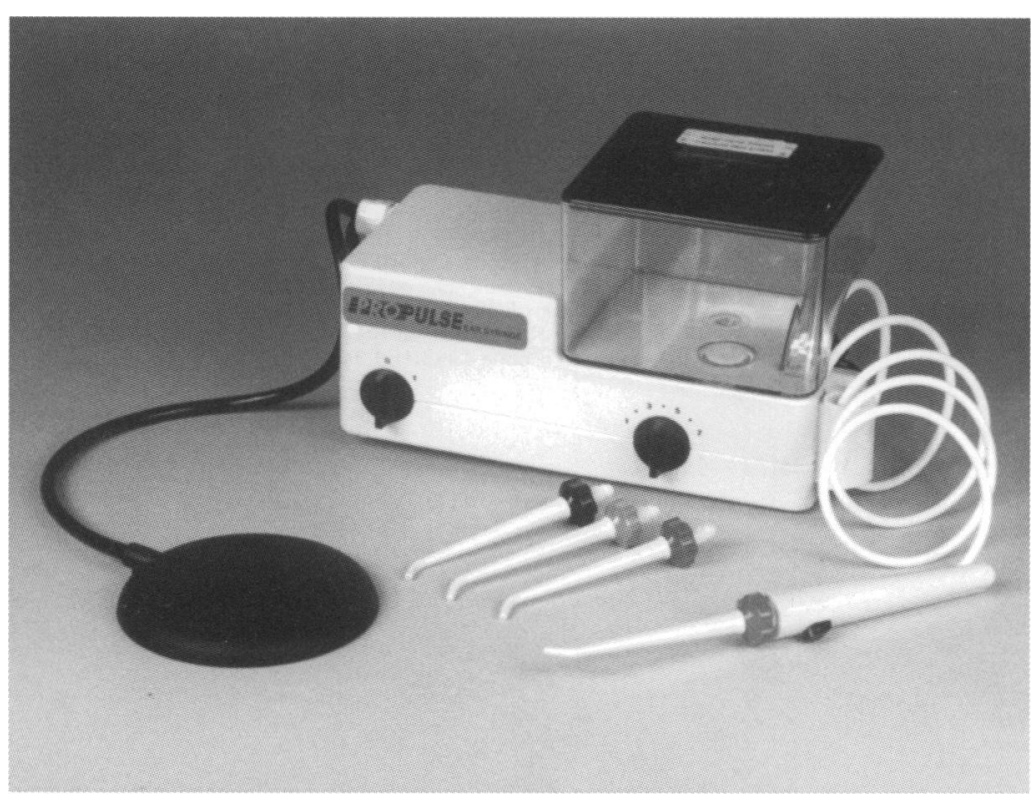

Figure 1 Propulse electronic pulsed ear syringe.
Patients with foreign bodies in the external auditory canal commonly present to an emergency department, accounting for approximately one in 500 paediatric and one in 1500 adult attendances. ${ }^{12}$ There are three standard techniques for the removal of these foreign bodies: (1) direct instrumentation (with Hartmann's crocodile forceps, hooks, or loops), (2) suction, and (3) irrigation.

Traditionally, the former method has usually been employed in the emergency department, although there are several problems with instrumentation, especially in children:

- Lack of cooperation from the patient.

- Difficulty with large, smooth, impacted foreign bodies.

- Inappropriately sized instruments.

- Potential for iatrogenic trauma. ${ }^{3-5}$

Ear, nose, and throat (ENT) surgeons generally consider suction removal using a microscope as the gold standard. ${ }^{6}$ However, this requires a general anaesthetic for children with consequent admission to hospital.

Irrigation is a long established method of removing non-hygroscopic foreign bodies from the auditory canal. Impacted wax (a common cause of impaired hearing) is usually removed in this way by general practitioners. In the UK, a variety of manual devices and one electric machine are available for syringing ears. The Propulse electronic pulsed ear syringe (Mirage, Bolton) is a patented instrument which costs just under $£ 100+$ tax (fig 1). It is widely used in the community and has been evaluated in our emergency department (which treats over 70000 new patients per annum) during the last three years.

\section{Methods and results}

Over a period of three years, an electric ear syringe was freely available for use in the emergency department by all doctors and nurse practitioners who had been trained in the technique of ear syringing (see table 1). For the second year of this period, a retrospective inspection was made of the records of all patients who presented with an aural foreign body.

The results from the one year audit are shown in table 2 . It is seen that:

- Almost $60 \%$ of all patients with foreign bodies in the auditory canal were children under the age of 16 years (and these were mostly preschool children).

- There was a 2:1 male to female ratio.

- Staff chose to treat over $50 \%$ of the patients with the electric ear syringe. 
Table 1 Instructions for the use of the electric ear syringe

- Explain the procedure to the patient

- Confirm the presence of a foreign body by auroscopy and identify any contraindications to irrigation

- Position the patient with the head tilted slightly towards the affected ear, a protective towel should be placed round the shoulders and a collecting tank positioned below the pinna

- Connect a clean jet tip applicator to the tubing of the electric syringe

- Fill the reservoir with prewarmed saline (at around $38-39^{\circ} \mathrm{C}$ )

- Direct the syringe nozzle into the reservoir and switch on the pump for 10-15 seconds to eliminate trapped air or cold saline

- Gently retract the pinna upwards and backwards to straighten the meatus (backwards only for children)

- Insert the nozzle into the external auditory canal and switch on the pump (either foot or hand control) on the lowest power setting

- Direct the saline stream along the roof of the auditory canal, towards the posterior wall (that is "five to" and "five past" clock positions for the right and left ears respectively). If in doubt, aim for the occiput

- Irrigate the canal until the foreign body appears and then reinspect the canal and the tympanic membrane. Ensure excess saline is removed under direct vision. The pulse pressure can be increased for resistant foreign bodies. If at any stage the patient experiences pain or dizziness, the procedure should be stopped

- The overall success rates for removal of foreign bodies were: electric syringe, $93 \%$ in adults and $88 \%$ in children and instrumentation, $68 \%$ in adults and $50 \%$ in children.

No complications were documented with either procedure.

\section{Discussion}

The Propulse electric ear syringe has been used in general practice and ENT clinics for a number of years but, as yet, has not been widely adopted by emergency departments. The principal use for all forms of ear syringe is the removal of wax and none has been designed specifically for the purpose of removing foreign bodies.

The manufacturers claim that the pulsatile delivery of fluid from the electric ear syringe prevents any build up of pressure in the meatus, thus minimising both patient discomfort and the potential for perforation of the tympanic membrane. They also claim that the electric syringe is easier to use and quicker than conventional manual syringes such as Russell's or Bacon's ear syringes.

Correct technique is important whatever device is used to irrigate the external auditory canal (see table 1) and warmed solutions are essential to prevent vestibular disturbance. ${ }^{5}$ Contraindications to syringing which must be excluded include: current tympanic perforation, evidence of previous perforation or scarring (that is weaker membrane), and presence of grommets (relative contraindication).

Irrigation is not recommended for hygroscopic or metallic foreign bodies, especially button batteries. Fortunately, children-who constitute the majority of cases-present with predominantly non-hygroscopic foreign bodies.

The procedure for ear syringing is minimally invasive, easy to teach, and effective. In this trial, the electric ear syringe was widely used by both
Table 2 Results of a one year audit of patients with aural foreign bodies in an emergency department

\begin{tabular}{llll}
\hline & $\begin{array}{l}\text { Adults } \\
(n=35)\end{array}$ & $\begin{array}{l}\text { Children } \\
(n=49)\end{array}$ & $\begin{array}{l}\text { Total } \\
(n=84)\end{array}$ \\
\hline $\begin{array}{l}\text { Male:female ratio } \\
\text { Type of foreign body: }\end{array}$ & $24: 11$ & $31: 18$ & $55: 29$ \\
$\quad$ Plastic & 2 & 20 & 22 \\
Cotton wool & 24 & 7 & 31 \\
Stone/metal & 0 & 11 & 11 \\
Paper/rubber & 5 & 3 & 8 \\
Insect & 3 & 3 & 6 \\
Other & 1 & 5 & 6 \\
Success of removal: & & & \\
Electric syringe & $18 / 18$ & $21 / 24$ & $39 / 42$ \\
$\quad$ Instrumentation & $11 / 12$ & $8 / 16^{\star}$ & $19 / 28$ \\
Removal not attempted & 5 & 10 & 15 \\
ENT referrals & $6 / 35$ & $20 / 49$ & $26 / 84$ \\
\hline
\end{tabular}

* One child had a failed removal with forceps and then a successful irrigation.

medical and nursing staff but, as can be seen from table 2, a proportion of staff preferred to continue using Hartmann's crocodile forceps or refer patients directly to the ENT clinic. This freedom of choice for the staff allowed some assessment of the acceptability of the new equipment but prevented randomisation of the patients. As such, no formal comparison of the efficacy of the two main techniques is possible. Nevertheless, the comparative safety of irrigation over instrumentation suggests that the former is the preferred method for the removal of aural foreign bodies in the emergency department. The electric ear syringe is a safe and effective device for this purpose but has not yet been compared with conventional manual syringes.

1 Baker D. Foreign bodies of ears and nose in childhood. Pediatr Emerg Care 1987;3:67-70.

2 Fritz S, Kelen G, Sivertson K. Foreign bodies of the external auditory canal. Emerg Med Clin North Am 1987;5: 183.

3 Brown AFT. Accident and emergency diagnosis and management. 3rd Ed. Oxford: Butterworth Heinemann, 1996: 297.

4 Morton RJ, Phillips BM. Accidents and emergencies in children. Oxford: Oxford University Press, 1992: 99.

5 Bull PD. Lecture notes on diseases of the ear, nose and throat. 8th Ed. Oxford: Blackwell Science, 1996: 30-3.

6 Bressler K. Ear foreign body removal: a review of 98 cases. Laryngoscope 1993;103:367-70. 